\title{
Modeling Bumble Bee Population Dynamics with Delay Differential Equations
}

\author{
H.T. Banks*, J.E. Banks ${ }^{@}$, Riccardo Bommarco ${ }^{+}$, \\ A. N. Laubmeier*, N. J. Myers*, Maj Rundlöf ${ }^{\dagger}$, and Kristen Tillman* \\ ${ }^{*}$ Center for Research in Scientific Computation \\ North Carolina State University \\ Raleigh, NC 27695-8212 USA \\ and \\ ${ }^{\circledR}$ Undergraduate Research Opportunities Center \\ California State University, Monterey Bay \\ Seaside, California 93955 \\ and \\ + Swedish University of Agricultural Sciences \\ Department of Ecology \\ 75007 Uppsala, Sweden \\ and \\ $\dagger$ Department of Biology \\ Lund University \\ 22362 Lund, Sweden
}

December 19, 2016

\begin{abstract}
Bumble bees are ubiquitous creatures and crucial pollinators to a vast assortment of crops worldwide. Bumble bee populations have been decreasing in recent decades, with demise of flower resources and pesticide exposure being two of several suggested pressures causing declines. Many empirical investigations have been performed on bumble bees and their natural history is well documented, but the understanding of their population dynamics over time, causes for observed declines, and potential benefits of management actions is poor. To provide a tool for projecting and testing sensitivity of growth of populations under contrasting and combined pressures, we propose a delay differential equation model that describes multi-colony bumble bee population dynamics. We explain the usefulness of delay equations as a natural modeling formulation, particularly for bumble bee modeling. We then introduce a particular numerical method that approximates the solution of the delay model. Next, we provide simulations of seasonal population dynamics in the absence of pressures. We conclude by describing ways in which resource limitation, pesticide exposure and other pressures can be reflected in the model.
\end{abstract}

Key words: population models, delay differential equations, non-linear, non-autonomous, spline approximations, Bombus terrestris, reproduction

\section{Introduction}

The protection of bumble bee populations, among other pollinators, is vital to sustain global agricultural food production $[29,43]$, biodiversity and ecosystem functioning $[28,50]$. It is now widely accepted that bumble bee diversity has dramatically declined in the past several decades $[10,15,16]$. Diminishing populations have been ascribed to habitat loss, resulting in loss of nest and flower resources, pathogens, climate change and exposure to chemical insecticides [56,64]. The buff-tailed bumble bee Bombus terrestris has been the subject of much study (see for example, $[1,23,24,24,52])$, as it is abundant in Europe and known to be an important pollinator [42]. Much 
experimental and analytic effort has been devoted to mapping its biology and natural history [14,34]. However, much less is understood about its population dynamics over time and the growth of bumble bee populations subjected to pressures and limitations of resources (see [20]).

Mathematical modeling based on empirical information on life history parameters can be a strong tool to project population dynamics and identify vulnerable traits and life stages, e.g., through sensitivity analysis [9, 21, 48]. With a realistic time-dependent model, it is possible to implement and study many suggested single and combined pressures that may affect bumble bees. Empirical research has concluded that forage resources (pollen and nectar) in the landscape affect overall bumblebee abundance. Furthermore, explicit modeling of resource dynamics over time has the potential to elucidate the mechanisms underlying these patterns and explain observed discrepancies (e.g., $[19,60,63,65]$ ) in which life stages (of queens, workers, males, and gynes) are supported under contrasting timing, amount, type and quality of food resources. Previous theoretical [51] and empirical [12] work has focused on the influence of particular aspects of foraging behavior and queen survival on colony growth. Special attention in particular has been given to modeling the allocation of resources among workers and sexual offspring and its implications for colony growth [51], with some evidence that optimal tradeoffs are a function of colony size as well as queen egg-laying rates $[11,55]$. We take a broader approach here, developing a population model in which we can explicitly test hypotheses about how landscape use and exposure to environmental toxins affect bumblebee populations.

We are motivated by the desire to understand the various ways in which $B$. terrestris populations are dynamically affected by environmental pressures, including pesticide exposure and resource limitation [32, 35, 46]. Mathematical modeling, especially in an iterative approach [9], can be used for projecting population abundance and understanding the importance of life history traits, such as survival, reproduction and seasonal reproductive switch times under contrasting scenarios. Mathematical modeling, particularly when paired with rich empirical data, provides analytic tools that experimentation alone cannot offer [7]. In this paper, we present a delay differential equation (DDE) model to simulate the abundance of different bumble bee castes and in-nest resources over time, with dynamics including colony establishment, mortality, colony growth, reproduction, and queen hibernation. Delay equations have been used in various applications, including biology, ecology, engineering (see $[2,22,33,38]$ for examples) and even honeybee population modeling [41]. We refer the reader to [61] for an introduction to DDEs and applications, as well as [44] for DDEs in ecology.

We present our model with the underlying assumptions, including a description of the literature references which provided us either direct or indirect estimates of some model parameters. We naturally introduce the class of DDEs and provide a brief overview for the reader. We introduce a linear spline approximation method for obtaining a numerical solution to our model. Next we provide model simulations in the absence of pressures. Lastly, we propose ways in which pressures such as resource limitation and insecticide exposure can be reflected in the model.

\section{Model}

\subsection{Our Proposed Model}

As we shall further develop below, our model is naturally a nonlinear system of delay differential equations (DDE) which describe six state variables in a collection of bumble bee colonies: in-nest nectar abundance $A(t)$, in-nest pollen abundance $B(t)$, queens $Q(t)$, workers $W(t)$, males $M(t)$ and gynes (daughter queens) $G(t)$. According to [31], only $70 \%$ of foragers return to their own colony after a foraging trip; we therefore assume that there is not-insignificant interaction between colonies and model a collection of bumblebee colonies which share a common pool of resources. While our model certainly allows for multiple year projections, we consider a time span of less than one year here. We define the first day of spring $T_{S}:=0$, which denotes the day on which all hibernating gynes emerge from hibernation to become queens and found new colonies. The independent variable $t$ measures time in days.

We consider the following assumptions and basic seasonal timeline $[14,24,34,49]$. Hibernating gynes emerge and become queens that found new colonies at $t=T_{S}$. These queens immediately begin foraging for and storing 
resources (nectar and pollen) inside the nest, as well as producing worker eggs. In the absence of information about queen foraging efficacy, we assume that the queen accumulates a negligible store of resources during this time, primarily providing for herself and the initial brood of workers. Assuming a 22-day worker incubation time (from an egg laid to the emergence of an adult worker) [24,34], the first workers emerge at $t=T_{S}+22$. At this time, the workers take over resource foraging to develop a store of in-nest resources and tending to new eggs, while the queens devote all energy to production of worker eggs [34]. The authors of [14,34] discuss in detail the somewhat mysterious process of bumble bee reproduction. There are varying theories on what factors contribute to the switch from worker to male and queen offspring production; these factors include, but are not limited to, queen condition during the season or during hibernation, queen pheromones, and worker abundance $[14,24,25,34,37,45,49,59]$. Environmental conditions can also cause nests to have either early or late season switch times [24]. In our model, we assume at some time $t=T^{*}$, the queen begins to lay sexual (male and gyne) eggs while continuing to produce worker eggs [14]. At time $t=T^{* *}$, the queen stops producing worker eggs and devotes all energy to sexual egg production. At time $t=T^{* *}+22$, the last new worker emerges. At times $t=T^{*}+26$, and $t=T^{*}+30$, respectively, the first males and gynes emerge (assuming respective 26 and 30 day incubation periods [24]). Sexuals continue to emerge until time $t=T_{W}$, at which point workers, queens and males die, and gynes go into hibernation and prepare to become queens in the following year $[14,34]$. The exact values of these timeline points depend greatly on geography, environment and weather. Furthermore, we believe that allowing $T^{*}$ and $T^{* *}$ to be functions of the worker population will allow us, in future work, to explore whether environmental changes and pressures such as insecticide exposure can have an indirect effect on reproductive switch times. A timeline of the bumble bee's seasonal life cycle is depicted in Figure 1. To demonstrate the usefulness of the DDE model, we fix timeline values $T^{*}, T^{* *}, T_{S}$ and $T_{W}$ at the values estimated by [49] as given in Table 1 and described in Section 2.2 below.

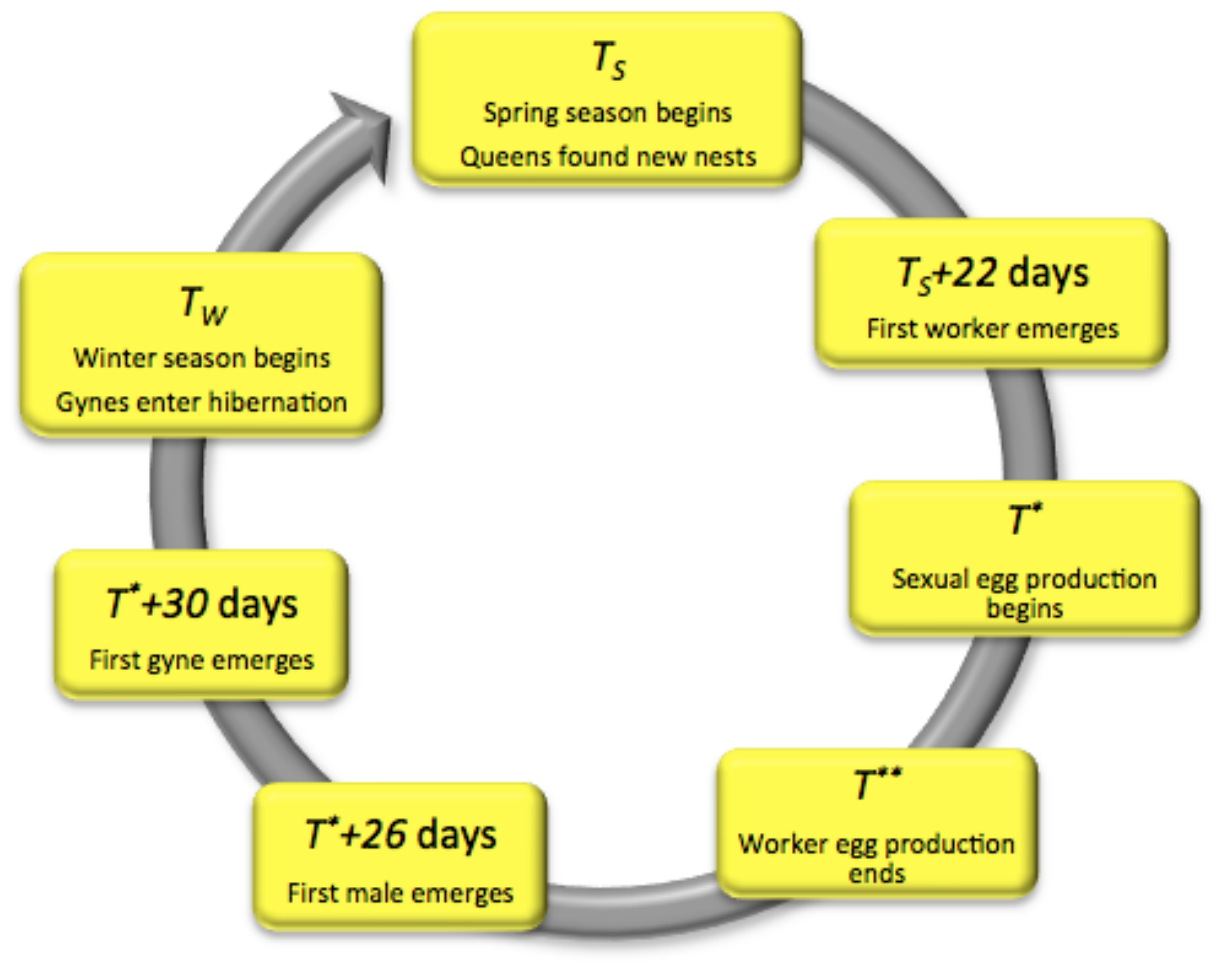

Figure 1: Timeline of bumble bee seasonal dynamics

We assume that the founding queen, workers, as well as worker, male and gyne larvae consume both nectar and pollen [24]. We explicitly assume that nectar and pollen consumption in the nest is negligible for adult males and adult gynes; [34] notes that males leave the colony a few days after reaching adulthood and that gynes may 
stay in the nest and forage for some time before finding a place to hibernate. In the absence of estimations for the resource consumption by emerging males and gynes in a colony, we assume that this short period of consumption is negligible and do not include it in the current model. We also assume that all colony members have a mortality rate, which encompasses mortality by aging and mortality during foraging trips. Although we assume that adult workers and queens consume resources, we do not model their mortality as a function of resources. We now present the mathematical model as well as the pre-spring history information in (1) and (2). Because of the previously described changes in seasonal dynamics, some differential equations are piecewise defined. All model variables, switch times, and parameters are described in Table 1. In addition, to elucidate the definitions of $\frac{d A}{d t}$ and $\frac{d B}{d t}$, we have included a description of seasonal resource (nectar and pollen) consumption and collection changes in Table 2. Resource consumption by larvae, given by the functions $l_{W}, l_{M}$, and $l_{G}$ in (1), and its functional dependence on $Q_{t}(a)=Q(t-a), a \in[\alpha, \beta]$ will be described later in more detail.

$$
\begin{aligned}
& \frac{d A}{d t}=\left(b_{A W}-\mu_{A W}\right) W-\mu_{A Q} Q-2\left(l_{W}\left(t, Q_{t}\right)+l_{M}\left(t, Q_{t}\right)+l_{G}\left(t, Q_{t}\right)\right) \\
& \frac{d B}{d t}=\left(b_{B W}-\mu_{B W}\right) W-\mu_{B Q} Q-\left(l_{W}\left(t, Q_{t}\right)+l_{M}\left(t, Q_{t}\right)+l_{G}\left(t, Q_{t}\right)\right) \\
& \frac{d Q}{d t}=-\mu_{Q} Q \\
& \frac{d W}{d t}=b_{W}(t-22) Q(t-22) \frac{1}{9} \int_{t-18}^{t-9} L\left(\gamma_{W} W(s)\right) L\left(\frac{A(s)+\frac{B(s)}{d_{B}}}{Q(s)\left(A_{\max }+\frac{B_{\max }}{d_{B}}\right)}\right) d s-\mu_{W} W \\
& \frac{d M}{d t}=b_{M}(t-26) Q(t-26) \frac{1}{11} \int_{t-22}^{t-11} L\left(\gamma_{M} W(s)\right) L\left(\frac{A(s)+\frac{B(s)}{d_{B}}}{Q(s)\left(A_{\max }+\frac{B_{\max }}{d_{B}}\right)}\right) d s-\mu_{M} M \\
& \frac{d G}{d t}=b_{G}(t-30) Q(t-30) \frac{1}{13} \int_{t-26}^{t-13} L\left(\gamma_{G} W(s)\right) L\left(\frac{A(s)+\frac{B(s)}{d_{B}}}{Q(s)\left(A_{\max }+\frac{B_{\max }}{d_{B}}\right)}\right) d s-\mu_{G} G \\
& A(t)= \begin{cases}0 & t<T_{S}+22 \\
A_{0} & t=T_{S}+22 \\
0 & t \geq T_{W}\end{cases} \\
& W(t)= \begin{cases}0 & t<T_{S}+22 \\
W_{0} & t=T_{S}+22 \\
0 & t \geq T_{W}\end{cases} \\
& B(t)= \begin{cases}0 & t<T_{S}+22 \\
B_{0} & t=T_{S}+22 \\
0 & t \geq T_{W}\end{cases} \\
& M(t)= \begin{cases}0 & t<T^{*} \\
M_{0} & t=T^{*}+26 \\
0 & t \geq T_{W}\end{cases} \\
& Q(t)= \begin{cases}0 & t<T_{S} \\
G\left(T_{S}-1\right) & t=T_{S} \\
0 & t \geq T_{W}\end{cases} \\
& G(t)= \begin{cases}G_{0} e^{-\mu_{G_{W}} t} & t<T_{S} \\
0 & t=T_{S} \\
G_{1} & t=T^{*}+30\end{cases}
\end{aligned}
$$




\begin{tabular}{|c|c|c|c|}
\hline Variable & Description & Units & Estimate \\
\hline$t$ & time & days & \\
\hline$A(t)$ & amount of nectar in colonies & $\mathrm{ml}$ & \\
\hline$B(t)$ & amount of pollen in colonies & $\mathrm{g}$ & \\
\hline$Q(t)$ & number of queens & individuals (queens) & \\
\hline$W(t)$ & number of workers & individuals (workers) & \\
\hline$M(t)$ & number of males & individuals (males) & \\
\hline$G(t)$ & number of gynes & individuals (gynes) & \\
\hline \multicolumn{4}{|l|}{ Timeline } \\
\hline$T_{S}$ & first day of spring & & $T_{S}:=0$ \\
\hline$T^{*}$ & first day male/gyne eggs laid & & $40[49]$ \\
\hline$T^{* *}$ & end of worker eggs laid & & $44[49]$ \\
\hline$T_{W}$ & beginning of winter & & 120 \\
\hline \multicolumn{4}{|l|}{ Parameters } \\
\hline$\mu_{A Q}$ & queen nectar consumption rate & $\frac{\mathrm{ml}}{\text { day-individual }(Q)}$ & 1 \\
\hline$\mu_{B Q}$ & queen pollen consumption rate & $\frac{\mathrm{g}}{\mathrm{day} \cdot \text { individual }(Q)}$ & 0.8 \\
\hline$\mu_{Q}$ & queen death rate & $\frac{1}{\text { day }}$ & $0.0154[30]$ \\
\hline$b_{A W}$ & worker nectar collection rate & $\frac{\mathrm{ml}}{\text { day.individual }(W)}$ & $0.6[36,52]$ \\
\hline$b_{B W}$ & worker pollen collection rate & $\frac{\mathrm{g}}{\text { day-individual }(W)}$ & $0.4[27,36]$ \\
\hline$\mu_{A W}$ & worker nectar consumption rate & $\frac{\mathrm{ml}}{\text { day-individual }(W)}$ & 0.15 \\
\hline$\mu_{B W}$ & worker pollen consumption rate & $\frac{\mathrm{g}}{\mathrm{day} \cdot \mathrm{individual}(W)}$ & 0.15 \\
\hline$b_{W}(t)$ & worker birth rate & $\frac{\text { workers }}{\text { queen.day }}$ & 2.4 \\
\hline$\mu_{W}$ & worker death rate & $\frac{1}{d a y}$ & 0.05 \\
\hline$\gamma_{W}$ & worker-worker larvae survival coefficient & $\frac{1}{\text { individual }(W)}$ & 0.0025 \\
\hline$b_{M}(t)$ & male birth rate & $\frac{\text { males }}{\text { queen.day }}$ & 1.2 \\
\hline$\mu_{M}$ & male death rate & $\frac{1}{\text { day }}$ & 0.01 \\
\hline$\gamma_{M}$ & worker-male larvae survival coefficient & $\frac{1}{\text { individual }(W)}$ & 0.0025 \\
\hline$b_{G}(t)$ & gyne birth rate & $\frac{\text { gynes }}{\text { queen } \cdot \text { day }}$ & 0.8 \\
\hline$\mu_{G}$ & in-season gyne death rate & $\frac{1}{d a y}$ & 0.01 \\
\hline$\mu_{G_{W}}$ & hibernation gyne death rate & $\frac{1}{\text { day }}$ & \\
\hline$\gamma_{G}$ & worker-gyne larvae survival coefficient & $\frac{1}{\text { individual }(W)}$ & 0.0025 \\
\hline$A_{\max }$ & in-nest nectar maximum (per nest) & $\frac{\mathrm{ml}}{\text { individual }(Q)}$ & 150 \\
\hline$B_{\max }$ & in-nest pollen maximum (per nest) & $\frac{g}{\text { individual }(Q)}$ & 150 \\
\hline$d_{B}$ & pollen grain density & $\frac{\mathrm{g}}{\mathrm{ml}}$ & $1[17]$ \\
\hline$P_{0}$ & initial larval pollen consumption & $\frac{\mathrm{g}}{\frac{\mathrm{g}}{\text { individual }(W) \cdot \text { day }}}$ & $0.001[57]$ \\
\hline $\bar{r}$ & $\begin{array}{c}\text { larval pollen consumption } \\
\text { growth rate }\end{array}$ & $\frac{1}{\text { day }}$ & $0.25[57]$ \\
\hline
\end{tabular}

Table 1: Model variables and parameters. The selected value for simulations is given in the last column; we include a source reference for values drawn from the literature. 


\begin{tabular}{|c|c|}
\hline Time interval & Resource collection and consumption \\
\hline$\left[T_{S}, T_{S}+22\right)$ & $\begin{array}{c}\text { queen collecting and consuming resources } \\
\text { worker larvae consuming resources after } T_{S}+4 \\
\text { negligible accumulation of in-nest resources }\end{array}$ \\
\hline$\left[T_{S}+22, T^{*}\right)$ & $\begin{array}{r}\text { workers collecting and consuming resources } \\
\text { queen consuming resources } \\
\text { worker larvae consuming resources }\end{array}$ \\
\hline$\left[T^{*}, T^{* *}+22\right)$ & $\begin{array}{r}\text { workers collecting and consuming resources } \\
\text { queen consuming resources } \\
\text { worker larvae consuming resources until } T^{* *}+13 \\
\text { male larvae consuming resources after } T^{*}+4 \\
\text { gyne larvae consuming resources after } T^{*}+4\end{array}$ \\
\hline$\left[T^{* *}+22, T_{W}\right)$ & $\begin{array}{c}\text { workers collecting and consuming resources } \\
\text { queen consuming resources } \\
\text { male larvae consuming resources until } T_{W}-11 \\
\text { gyne larvae consuming resources until } T_{W}-13\end{array}$ \\
\hline
\end{tabular}

Table 2: Seasonal time intervals and corresponding effects of colony members on resources, nectar and pollen.

\subsection{Model and Parameter Considerations}

For our simulations, additional model and parameters considerations were necessary. We searched the literature to determine reasonable values for various model assumptions and parameters. Other parameters in the model, such as the death rate of adult bees, are difficult to empirically assess in natural environments. Even parameters for which a measured value can be found in the literature may be scenario dependent and expected to vary between applications. For these remaining parameters, we select values which are feasible in the defined units and, for the purpose of demonstrating the interplay between resources, workers, and sexual bees, do not result in total population loss. In Table 1 all model variables, time points, parameters and initial conditions are reported with corresponding units and literature comments. We now comment further on these choices.

The function $L$ used at several places in (1) is defined by

$$
L(x)=\frac{1-e^{-x}}{1+e^{-x}} .
$$

This is an increasing, saturating sigmoid function depending on the argument $x$ (much like a cumulative distribution function to describe probability of survival) [41]. The function $L$ is bounded in [0,1] and used to describe various daily survival rates of worker, male and gyne larvae, where $x$ depends on the population of worker bees which tend to the larvae or in-nest resources.

In our model equations for workers, males and gynes, we see the term $A(s)+d_{B}^{-1} B(s)$, quantifying the net resources at time $s$. Because $A$ is measured in milliliters, and $B$ is measured in grams, it is reasonable to use a unit of either $\mathrm{ml}$ or $\mathrm{g}$, so without loss of generality, we choose the unit $\mathrm{ml}$. We can convert the mass of pollen $B$ into volume using the density of pollen. In other words,

$$
B_{V}(t)=d_{B}^{-1} B(t)
$$

where $B_{V}(t)$ denotes the volume of pollen and $d_{B}$ denotes its density (a constant). There are various species of pollen, each with different specific material densities. According to [17], an estimated value for pollen grain density is $1 \mathrm{~g} / \mathrm{ml}$. So we choose $d_{B}=1$ for the preliminary simulations.

When modeling resource consumption we use the fact that a bumble bee goes through three sequential stages: egg, larval, and pupal before becoming an adult. Larvae consume resources but eggs and pupa do not; we note the effect this has on seasonal resource consumption in Table 2. Larval stage length can be modified based on 
food availability [62] or in-nest temperature. For simplicity, we assume that bumble bees have constant incubation schedules, seen in Table 3 [24].

\begin{tabular}{|c|c|c|c|c|}
\hline Caste & Total no. incubation days & Egg (days) & Larva (days) & Pupa (days) \\
\hline Workers & 22 & 4 & 9 & 9 \\
\hline Males & 26 & 4 & 11 & 11 \\
\hline Gynes & 30 & 4 & 13 & 13 \\
\hline
\end{tabular}

Table 3: Bumble bee incubation breakdown by caste

Our model tracks resources (pollen and nectar) and adult colony members (queens, workers, males and gynes). However, all larvae contribute to resource consumption as well and this dynamic must be included in the model. In Table 2, we summarize the effects of all colony members on resources at all phases of the season. We tentatively assume, for sake of simplicity, that the last worker emerges when the first male emerges (because of our knowledge of incubation times of 22 and 26 days for workers and males, respectively, and our estimate that $T^{*}=40$ and $T^{* *}=44$, as seen in Table 1). With these assumptions, we have three functionals $l_{W}\left(t, Q_{t}\right), l_{M}\left(t, Q_{t}\right)$ and $l_{G}\left(t, Q_{t}\right)$, in (1), which reflect larval pollen consumption, which require definition.

According to $[54,57,58]$ daily pollen intake (measured in mass of pollen per larvae per day) increases exponentially as age increases. Therefore, we can model daily pollen intake per worker larvae by $P_{0} e^{\bar{r} a}$ where $a$ measures age with unit [day], for some positive constants $P_{0}$ and $\bar{r}$. The expected number of worker larvae of age $a$ at time $t$ is given by $b_{W}(t-a) Q(t-a)$, for $4 \leq a \leq 13$. Therefore, we can model pollen consumption per day by worker larvae as

$$
l_{W}\left(t, Q_{t}\right)=\int_{4}^{13} b_{W}(t-a) Q(t-a) P_{0} e^{\bar{r} a} d a .
$$

A simple change of variables can be used to express this in terms of hysteretic dependence on $Q$ given by

$$
l_{W}\left(t, Q_{t}\right)=\int_{t-13}^{t-4} b_{W}(s) Q(s) P_{0} e^{r(t-s)} d s .
$$

Note that this functional $l_{W}\left(t, Q_{t}\right)$ depends on the values of $Q(s), t-13 \leq s \leq t-4$. Similarly, we have

$$
l_{M}\left(t, Q_{t}\right)=\int_{4}^{15} b_{M} Q(t-a) P_{0} e^{\bar{r} a} d a,
$$

for pollen consumption by male larvae which can be written as

$$
l_{M}\left(t, Q_{t}\right)=\int_{t-15}^{t-4} b_{M}(s) Q(s) P_{0} e^{r(t-s)} d s,
$$

and

$$
l_{G}\left(t, Q_{t}\right)=\int_{4}^{17} b_{G} Q(t-a) P_{0} e^{\bar{r} a} d a
$$

for pollen consumption by gyne larvae which can be written as

$$
l_{G}\left(t, Q_{t}\right)=\int_{t-17}^{t-4} b_{G}(s) Q(s) P_{0} e^{r(t-s)} d s
$$

We note that the functional dependence on $Q(s)$ is different in each of $(3),(4)$ and (5). We will however simply denote the dependence by $Q_{t}$ in a slight abuse of notation which should not result in confusion for the reader.

By examining data in [57], we assume that initial larval consumption (per larva) $P_{0}$ is constant across castes, along with exponential consumption rate $\bar{r}$. In addition, according to [53], larval diet consists of approximately $34 \%$ pollen, and the rest is a combination of nectar and a trivial amount of digestive enzymes. Consequently, we can assume an approximate 2:1 ratio of nectar to pollen for larval consumption, which explains the larval consumption 
terms in the model for $\frac{d A}{d t}$. We note, however, that the functionals $l_{W}, l_{M}$, and $l_{G}$ assume consumption by larvae with total survivability into adulthood, while (1) assumes some proportion of all larvae do not survive to adulthood. Our model underestimates the store of in-nest resources available to hives by overestimating the consumption of these resources by larvae. Furthermore to capture the first brood $\left(W_{0}\right)$ dynamics of the colony [24], the first brood is assumed to have the necessary resources and size to grow to adulthood, while larval consumption (and the necessary resource collection) controls the hive dynamics for all larvae after the initiation of a queen's first brood.

Since different colonies within a region can exhibit different growth and reproductive strategies [34], the initial conditions in (2) may be non-zero valued in order to capture the appropriate dynamics. To accurately simulate first brood dynamics, the initial condition $W_{0}=192$ accounts for the number of workers produced at $T_{S}+22[24]$ corresponding to the number of beginning colonies, $G_{0}=20$. Similarly, $M_{0}$ and $G_{1}$ allow for large emergence of males and gynes respectively. The non-zero initial values $A_{0}$ and $B_{0}$ may represent in-nest stores of pollen and nectar which remain after the queen rears the first brood. As indicated previously, we assume no in-nest stores when workers emerge at $T_{S}+22$. We also assume no large emergence of males and gynes, taking $M_{0}, G_{1}=0$.

In addition, we simplify the model by letting the worker, male and gyne birth rates $b_{W}(t), b_{M}(t)$, and $b_{G}(t)$ respectively, be time dependent expressions given by

$$
\begin{aligned}
& b_{W}(t)= \begin{cases}\bar{b}_{W} & T_{S} \leq t \leq T^{* *} \\
0 & \text { else }\end{cases} \\
& b_{M}(t)= \begin{cases}\bar{b}_{M} & T^{*} \leq t \leq T_{W}-26 \\
0 & \text { else }\end{cases} \\
& b_{G}(t)= \begin{cases}\bar{b}_{G} & T^{*} \leq t \leq T_{W}-30 \\
0 & \text { else }\end{cases}
\end{aligned}
$$

This allows for proper simulation of the phases described in Table 2 by equations (3) - (5).

To determine the seasonal switch times $\left(T^{*}, T^{* *}\right)$, we use the following information taken from [49]. Let the term "first egg" denote the first egg laid in the colony, regardless of caste determination. In the experiments conducted in [49], the average time from first egg to last worker emergence was approximately 66 days; assuming a 22 day incubation time, this implies that $T^{* *}=44$. The average time from first egg to first emergence of gynes and males was 70 and 65 days respectively. Assuming 30 and 26 day incubation periods for gynes and males, respectively, this provides two estimates for $T^{*}: T^{*}=40$ or $T^{*}=39$. Because these values are so similar, we assume $T^{*}=40$ for our simulations.

Also from the literature, values for $b_{W}$ and $\mu_{Q}$ were determined; the reference [49] provides an estimate for the average number of workers produced per day in a given colony, which gives us a direct estimate: $b_{W}=2.4$. Then by [30], we used the information that 14 out of 40 total colonies saw mother queen loss (hence 26 queens survived) in a given experiment lasting 28 days. We performed a simple inverse problem for the model

$$
\frac{d Q}{d t}=-\mu_{Q} Q, \quad Q(0)=40
$$

with the sole data point $Q(28)=26$. This returns the best fitting estimate $\mu_{Q}=0.0154$. We initially use these values in our simulations.

Last, we note that the state variables may be properly viewed as components of the $6 \times 1$-vector $\mathbf{x}$ satisfying delay differential equations $[2-4,6,8,22,33,39,40,61]$ which have been widely used over the last several decades in population models. As is known, these delay equations require not only the initial values, $\eta$, of the states at time $0\left(t \equiv T_{S}\right)$, but also the history information, $\phi$ for all $t \in[-\bar{\tau}, 0)$, for $\bar{\tau}$ defined by the model (in this case, $\bar{\tau}=30$ ). For now, we further simplify the model by changing the history information for the gyne population, $G$. Instead of the exponential decay we see in (2) for $t<T_{S}$, we will first assume that $G$ remains a non-zero constant value $G_{0}$ for $t<T_{S}$. Initially, we made these choices of history information,

$$
\eta=\left[A_{0}, B_{0}, Q_{0}, 0,0,0\right]^{T}
$$


where $Q_{0}=G\left(T_{S}-1\right)=G_{0}$ (i.e., the number of queens when spring begins is the number of gynes at $t=-1$, i.e., at the end of last season), and

$$
\phi=\left[0,0,0,0,0, G_{0}\right]^{T},
$$

for some constants $A_{0}, B_{0}$, and $G_{0}$. With these choices of history information, all history functions are continuous except at $t=0$ where there is a jump discontinuity in four of the six variables. While this is theoretically acceptable for our numerical solution method of using spline approximations (discussed briefly below and in more detail in [5]), in practice this jump discontinuity creates a computational error in our model that is propagated over time (because solving delay equations requires iteratively solving large linear systems, which we describe in detail in [5]). To alleviate this, we instead chose a continuous history function by incorporating "ramp" functions for nectar, pollen, queens, and gynes. For example, let $\phi_{A}(t)$ and $\eta_{A}$ denote the history function on $t \in[-\bar{\tau}, 0)$ for nectar and $A(0)$, respectively. Then we have

$$
\phi_{A}(t)=\left\{\begin{array}{ll}
0 & -\bar{\tau}<t<-2 \\
\eta_{A}\left(\frac{t}{-2}+1\right) & -2 \leq t<0
\end{array} .\right.
$$

Defining similar ramp functions and incorporating them into our history for pollen, gynes and queens alleviates the computational error faced with the jump discontinuities at $t=0$.

\subsection{Sensitivity Methods}

With the above parameters, we investigate the sensitivity of our model to parameters $b_{A W}, b_{B W}, \gamma$, and $W_{0}$ by computing the population values of the collection of colonies at the end of a single season across a range of parameter values. The number of gynes and males produced by the end of a season are an indicator of the colonies' reproductive viability, and so we are particularly interested in how these values are affected by the model parameters. The choice of parameters and corresponding ranges are explained below.

The parameter for the nectar collection rate of worker bees, $b_{A W}(\mathrm{ml} /$ day/individual) takes values in the interval $[.4,1]$ and for our main simulation is fixed at $b_{A W}=.6$. According to $[36,52]$, nectar foraging efficiency of worker bees fall on average in the range $[0,9.6]$ (g/day). From [36], we assume that at any time, $1 / 3$ of all worker bees are foraging and [52] reports that the ratio of pollen to nectar collecting foragers varies with time, with a near-equal split in the middle of the day and greater nectar collection near the ends of the day. In future work, we could consider time-varying nectar collection rates. For this initial sensitivity analysis, we assume that half of all foragers collect nectar. We therefore obtain nectar collection rates in the range $[0,1.6](\mathrm{ml} /$ day/individual). A smaller range of these possible values, $[.4,1]$ (ml/day/individual) is taken since we expect that collection rates at the population level will fall somewhere near the average of the observed range of collection rates. Values below .4 are excluded since they produce biologically implausible results.

The second parameter for consideration is the pollen collection rate of work bees, $b_{B W}$ (g/day/individual). The parameter $b_{B W}$ is taken from $[.35, .6]$ and for our main simulation is fixed at $b_{B W}=.4$. We estimate these ranges from observations in $[27,36]$, where pollen foraging efficiency of worker bees fall on average in the range $[0,3.6]$ (g/day). Using the same assumptions on worker foraging proportions for nectar, we obtain pollen collection rates in the range $[0, .6]$ (g/day/individual). Again, a smaller range of these possible values, [.35,.65]] (g/day/individual) is taken since we expect that collection rates at the population level will fall somewhere near the average of the observed range of collection rates. Values below .3 are excluded since they produce biologically implausible results.

We assume in our preliminary results that the worker-larvae survival coefficients, $\gamma_{W}, \gamma_{M}$, and $\gamma_{G}$, are all the same constant $\gamma$. The worker-larvae survival coefficient relates workers tending the nest to the survival of larvae. We take values of $\gamma \in\left[5 \times 10^{-5}, .5\right]$ and for our main simulation fix $\gamma=.0025$. The saturating probability function $L(x)$ satisfies $L(x) \geq .999$ for all $x \geq 10$. In $(1)$, we have $L(\gamma W(s))$. By letting $W_{\max }$ be the maximal number of workers that a hive needs to care for all larvae and require that $\gamma W_{\max } \geq 10$. This guarantees that $L\left(\gamma W_{\max }\right) \approx 1$ (i.e., that no larvae die due to worker neglect when the worker population is at $\left.W_{\max }\right)$ and that $L(\gamma W(s)) \leq 1$ for $W(s) \leq W_{\max }$.

From [34], we have that 350 is the largest worker population for a single colony. Since we begin our simulation with 20 colonies, we take $W_{\max }=7000$ to account for total bees in all the colonies and obtain $\gamma=10 / 7000 \approx .0014$. 
Although we find that $\gamma=.0014$ satisfies the condition that $\gamma W_{\max } \geq 10$, queens die throughout the season and we do not expect that the maximum number of workers are required to care for all larvae in an average hive. Therefore, we round to $\gamma=.0025$ and test the range $\gamma \in\left[5 \times 10^{-5}, .5\right]$ in order to simulate regions where larval survival has a stronger $(\gamma<.0025)$ or weaker $(\gamma>.0025)$ dependence on the number of workers in the hive.

The final parameter which represents the size of the first worker brood, $W_{0}$ (individual) takes values of $W_{0} \in$ $[130,410]$ and for our main simulation is fixed at $W_{0}=192$. The initial brood of workers for a single queen on average is 9.6 according to [24]. Using 20 colonies in our simulation, we assume that the initial brood of workers across all colonies is $W_{0}=192$. We test the range $[130,410]$ in order to simulate regions where the queens produce smaller (6.5 workers per colony) and larger (20.5 workers per colony) initial broods.

\section{Delay Differential Equations and Numerical Methods}

The discussion above led naturally to a vector system of delay differential equations which readily exhibit hysteresis effects in the bumble bee (as well as other populations) colony dynamics. We discuss briefly aspects of such systems by considering a scalar version of a typical DDE system. We consider the following parameter $(q)$ dependent DDE

$$
\begin{aligned}
& \frac{d x}{d t}(t)=f\left(t, x(t), x_{t}, x\left(t-\tau_{1}\right), \ldots, x\left(t-\tau_{\nu}\right), q\right)+g(t), \quad 0 \leq t \leq T, \\
& x_{0}(\theta)=\phi(\theta), \quad \theta \in[-\bar{\tau}, 0],
\end{aligned}
$$

where $f=f\left(\eta, \psi, y_{1}, \ldots, y_{\nu}\right): Z \times \mathbb{R}^{\nu} \rightarrow \mathbb{R}^{1}$. We define $Z=\mathbb{R}^{1} \times L_{2}\left(-\bar{\tau}, 0 ; \mathbb{R}^{1}\right), 0<\tau_{1}<\cdots<\tau_{\nu}=\bar{\tau}$, $x_{t}(\theta)=x(t+\theta)$ for $-\bar{\tau} \leq \theta \leq 0$ and $\phi \in H^{1}(-\bar{\tau}, 0)$. The function $g(t)$ can be understood as some control function (in the case of our initial model, $g(t) \equiv 0)$. The Banach space $L^{2}\left(a, b ; \mathbb{R}^{1}\right)$ is the space of all real-valued square integrable functions while $H^{1}$ is the space of absolutely continuous functions $f:[a, b] \rightarrow \mathbb{R}^{1}$ such that $\partial f=\dot{f} \in L_{2}\left(a, b ; \mathbb{R}^{1}\right)$. The natural state space for this system $[5,6,8]$ is an infinite dimensional function space such as $Z$. All theoretical and computational approximations (necessitated by the infinite dimensionality of the state space) to treat our system of DDE are discussed in detail in [5]. Here we simply provide brief comments on the numerical methods used to approximate the solution to (1)-(2). As mentioned previously, DDEs (sometimes called functional differential equations) differ from ordinary differential equations (ODEs) in many ways, including the dimension of the solution. Because (1)-(2) cannot be solved analytically using tools such as the method of steps [13], we must use some type of numerical method to find a finite dimensional approximation to the infinite dimensional solution. Matlab's built-in DDE solver, dde23, which uses a multi-step method to approximate the solution to a simple DDE with constant delays, is convenient and useful, but limited in scope. One main limitation is the solver's inability to handle continuum hysteresis terms, which are seen throughout our model, in functions $l_{W}, l_{M}$, and $l_{G}$ as well as the integral terms seen in equations for $\frac{d W}{d t}, \frac{d M}{d t}$ and $\frac{d G}{d t}$. In addition, our model is piecewise defined, and using the solution (output) of one DDE as the history "function" for a future DDE becomes quite clumsy with dde23. Therefore, it becomes useful to consider an approximation in some finite dimensional solution subspace, similar to the method of finite elements for partial differential equations. There are many possible choices of solution spaces, one of which is some space of spline functions $[2,4,8,39,40]$. While the use of cubic and other higher order spline methods can be considered, the authors of [8] found that piecewise linear splines, when implemented with a fairly fine mesh, produced excellent results. We found, as reported in detail in [5], similar computational results for our system (1)-(2).

\section{Results}

In Figure 2 we plot the solution to the system with the default parameter set described in Table 1. In the collection of colonies, the total number of workers decreases throughout the season, with the slowest rate of decrease occurring between days 40 and 60. The workers enter a phase of purely exponential decay towards the end of the season, driven by our definition of the period in which new workers are produced. The males and gynes both experience growth, but this growth is slow and levels off quickly. Resource levels increase significantly after workers emerge, 
with pollen growing at a slower rate than nectar by choice of parameters. In the colonies, the nectar and pollen resources level off toward the end of the season.
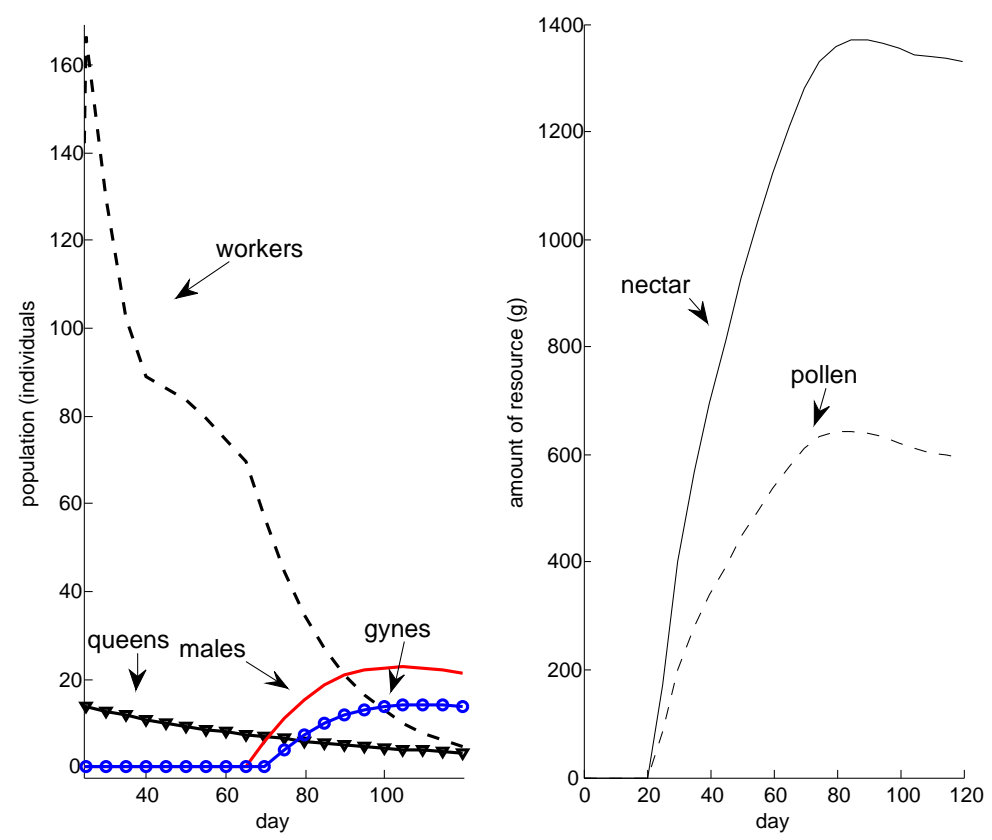

Figure 2: Population levels simulated with the default parameter set. Bee castes are plotted in the left graphs and resource levels are plotted in the right graphs.

In Figure 3 we plot the final population levels of the workers, males, and gynes as functions of parameters $b_{A W}$ (worker nectar collection rate), $b_{B W}$ (worker pollen collection rate), $\gamma$ (worker to larvae survival coefficient), and $W_{0}$ (size of initial worker brood). In Figure 4 we plot the final nectar and pollen levels as functions of the same parameters.

The final worker, male, and gyne populations do not appear to be sensitive to $b_{A W}$ or $b_{B W}$. However, these parameters have significant impacts on the amount of nectar and pollen available to bumblebee larvae; the final amount of nectar increases with $b_{A W}$ and, similarly, the final amount of pollen increases with $b_{B W}$.

The final level of all populations and resources share a positive, saturating relationship with $\gamma$. We note that for $\gamma=.4$, we have $L(\gamma W) \geq .999$ for all $W \geq 25$. That is, we have almost no larval mortality due to worker neglect while $W \geq 25$. Since $W_{0}=192$, there are sufficient worker bees for much of the season to guarantee that $L(\gamma W) \geq .999$. It is not surprising that increasing the value of $\gamma$ beyond this value does not significantly affect the final state of the system.

All states share a positive, linear relationship with $W_{0}$. We note that the final worker population does not exhibit high sensitivity to any of the tested parameters. This is not surprising; because workers enter a phase of exponential decay around $t=70$, they may be highly sensitive to the parameters, but this effect is diminished by the end of the season. 

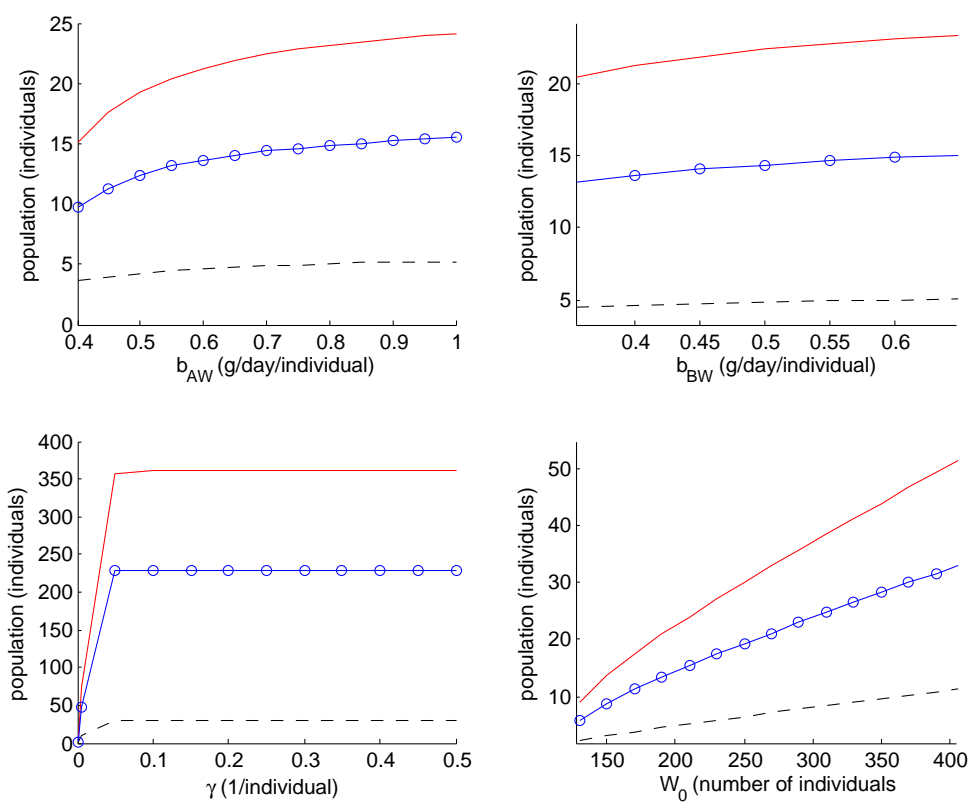

Figure 3: Sensitivity of the final populations of workers, males, and gynes to changes in $b_{A W}, b_{B W}, \gamma$, and $W_{0}$.
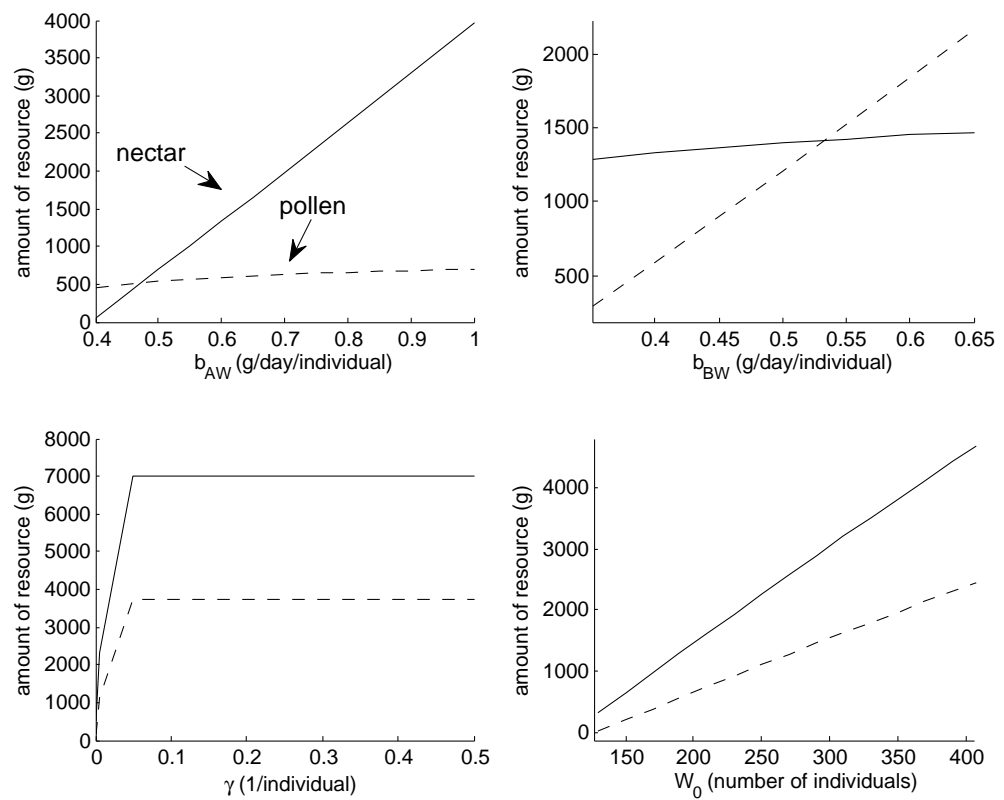

Figure 4: Sensitivity of the final nectar and pollen levels to changes in $b_{A W}, b_{B W}, \gamma$, and $W_{0}$.

\section{Discussion}

In this paper, we present a delay differential equation model to describe population dynamics of a collection of bumble bee colonies. Following [20], we place significant importance on the colonies' collection of nectar and pollen in determining larval survival and hive dynamics. We chose the natural use of delay equations to accurately capture the incubation times and dependence on the history of resource availability in bumble bee birth rates. We present the solutions to the model in a season where a lack of resources does not cause significant environmental pressure and the switch to production of bees occurs at a fixed time, independent of bee populations.

Without data, we cannot conclude that our model captures the behavior of a particular collection of bumble bee colonies. We find that compared to other theoretical works, our model performs adequately in describing bumble 
bee dynamics. In [20], total colony weight was expected to increase exponentially and subsequently decrease once workers begin to die and sexuals begin to leave the nests. Without modelling subadult stages, we cannot quantify colony weight for a direct comparison; we do note, however, that prior to the emergence of sexuals we see rapid growth of in-nest resources, measured in grams. Rates of birth into subadult classes are assumed to be constant, but we cannot draw conclusions about larval contributions to hive weight due to assumed mortality and adult emergence. Furthermore, queen production in [20], as in our model, was driven by multiple mechanisms, including floral resources. The general trend of increased total population until worker death and sexual dispersal seen in the model and experimental data of [20] lended further support to our proposed model, though Poitrineau et al. and others emphasize that this may be mitigated by worker efficiency and queen egg-laying rates $[11,55]$.

Our simulations capture late-season death of workers and the emergence of sexual bees, although nectar and pollen levels do not decrease late in the season, making it unlikely that colony weight decreases as observed in [20]. This is far from an optimal strategy for hive consumption of resources and may be an artifact of uncertainty in our parameters instead of a likely population dynamic; it is necessary in future work that we corroborate model output with available data in order to calibrate our assumed parameters for resource collection and consumption. Due to the manual selection of critical model parameters and the inherent uncertainty in those taken from the literature, we do not necessarily conclude that the model cannot replicate this weight pattern. Instead, we must in future efforts better estimate model parameters in order to match this classically assumed behavior.

The authors of $[47,55]$ model bumblebee reproductive switch times by optimal strategies; as explained in our model development, we assume an overlapping switch in which queens lay worker and sexual eggs simultaneously. Our choice of reproductive strategy is in line with the behaviors modelled in [55], but our model's assumed incubation times for sexual bees results in dynamics similar to those in [47], where the worker population is in complete decline when sexuals begin to emerge. With data, we can calibrate model parameters to obtain resource and population outputs at physically reasonable levels.

We can see in the sensitivity results that the size of the first brood of workers has a more significant impact on the male population than the gyne population. This may be affected by the birth rate of the males, which is higher than the gyne birth rate. However, the fact that males have a shorter development time (4 days less) may also enhance this effect; the history that affects an emerging male bee at time $t$ overlaps with, but is distinct from, the history that affects a gyne emerging at that same time. These types of delay dynamics are uniquely captured by our choice of model. Although the use of a DDE produced a more complex mathematical and computational problem to solve, we suggest that an ODE-based model would be insufficient to describe seasonal bumble bee population dynamics in this way.

The results from the sensitivity analysis also indicate that changes need to be made to the model in future simulations. We would expect that parameter values which can cause low levels of nectar or pollen in the hive (such as $b_{A W}$ and $b_{B W}$ ) would have a significant impact on larval survivability and therefore the final populations of males and gynes, but our model does not capture this behavior. This may be caused by considering the quantity $A+B / d_{B}$ in larval survivability; even when pollen resources are near zero, larval survivability can be high if nectar resources are sufficiently high. At the default parameter set presented here, resources are plentiful and the lack of resource-dependent mortality does not play a significant role. Resource scarcity also has no effect on adult mortality in the current model, which may contribute to the lack of sensitivity to $b_{A W}$ and $b_{B W}$. However, at extremely low resource levels, a persistent worker bee population may cause a rebound effect for resources, where total colony collapse would be more realistic. We note that the conflation of uncertainty in model assumptions and parameters makes it difficult to determine a single cause of model dynamics.

As mentioned above, it is important to understand bumble bee population dynamics in the presence of various environmental pressures, including resource scarcity and insecticide exposure. There are multiple ways to incorporate both lethal and sub-lethal effects of insecticide-exposure into population models of this sort, including changes in worker death rate, worker collection rate, worker productivity rates, queen death rate, and queen reproduction rates. In [18] the authors show that varying parameterizations of an ODE model can result in colony loss under sub-lethal stresses; in future work, we plan to experiment with similar parameters to obtain model predictions in the presence of environmental pressures. In particular, we may define the above parameters as functions of insecticide exposure, rather than as constants. In addition, one can implement changes due to environmental 
effects by exploring variable reproductive switch times, maximum resource availability, etc. By comparing model results to experimental data in the absence and presence of these pressures, one can test the model's accuracy.

\section{Conclusion}

Our results indicate that our model holds high promise for better understanding bumble bee population dynamics, especially in response to anthropogenic disturbance. A great challenge in terms of its predictive power is the accurate estimation of multiple unknown parameter values. For parameter estimation and subsequent model validation, we intend in our future work to use experimental longitudinal data tracking colony fitness, worker production, mother queen survival, and worker resource collection which are experimentally impacted by a particular pressure, such as both unexposed and neonicotinoid-exposed colonies, e.g., as in [26]. After using this data to estimate more parameters with a reasonable level of confidence [7], we will move to a motivating problem of understanding how pressures such as neonicotinoid exposure may have lethal and/or sublethal effects on bumble bee colonies. Some effects to be explored may include birth rates, resource consumption, worker productivity (efficient care of eggs/larvae), death rates, and season switch times. In addition, we would like to extend our simulations to include multi-seasonal projections to better understand long term effects of single and combined pressures such as habitat loss, resource limitations and pesticides.

\section{Acknowledgements}

We thank Oliver Schweiger for assisting with parameter values collected from the literature. We extend profuse thanks to the anonymous reviewers, whose comments greatly improved the manuscript. This research was supported in part by the Air Force Office of Scientific Research under grant number AFOSR FA9550-15-1-0298, in part by the National Science Foundation under Research Training Grant (RTG) DMS-1246991, in part by a CRSC/Lord Fellowship, by the August T. Larsson guest researchers programme at the Swedish University of Agricultural Sciences, and by the Swedish research council FORMAS.

\section{References}

[1] B. Baer and P. Schmid-Hempel. Sperm influences female hibernation success, survival and fitness in the bumble-bee Bombus terrestris. Proceedings of the Royal Society of London B: Biological Sciences, 272(1560):319-323, 2005.

[2] H.T. Banks. Delay systems in biological models: approximation techniques. Nonlinear Systems and Applications : 21-38, 1977.

[3] H.T. Banks. Approximation of nonlinear functional differential equation control systems. Journal of Optimization Theory and Applications, 29(3):383-408, 1979.

[4] H.T. Banks. Identification of nonlinear delay systems using spline methods. Nonlinear Phenomena in Mathematical Sciences : 47-55, 1980.

[5] H.T. Banks, J.E. Banks, R. Bommarco, A.N. Laubmeier, N.J. Myers, M. Rundlöf, and K. Tillman. Analysis of nonlinear delay systems with applications in bumble bee population models. CRSC-TR16-13, N.C. State University, Raleigh, NC, December, 2016, to appear.

[6] H.T. Banks and J.A. Burns. Hereditary control problems: numerical methods based on averaging approximations. SIAM Journal on Control and Optimization, 16(2):169-208, 1978.

[7] H.T. Banks, S. Hu, and W.C. Thompson. Modeling and inverse problems in the presence of uncertainty. CRC Press, 2014. 
[8] H.T. Banks and F. Kappel. Spline approximations for functional differential equations. Journal of Differential Equations, 34(3):496-522, 1979.

[9] H.T. Banks and H.T. Tran. Mathematical and experimental modeling of physical and biological processes. CRC Press, 2009.

[10] I. Bartomeus, J.S. Ascher, J. Gibbs, B.N. Danforth, D.L. Wagner, S.M. Hedtke, and R. Winfree. Historical changes in northeastern US bee pollinators related to shared ecological traits. Proceedings of the National Academy of Sciences, 110(12):4656-4660, 2013.

[11] M. Beekman, R. Lingeman, F.M. Kleijne, and M.W. Sabelis. Optimal timing of the production of sexuals in bumblebee colonies. Entomologia Experimentalis et Applicata, 88(2):147-154, 1998.

[12] M. Beekman, P. Stratum, and R. Lingeman. Diapause survival and post-diapause performance in bumblebee queens (Bombus terrestris). Entomologia Experimentalis et Applicata, 89(3):207-214, 1998.

[13] A. Bellen and M. Zennaro. Numerical methods for delay differential equations. Oxford university press, 2013.

[14] T. Benton. Bumblebees: the natural history $\&$ identification of the species found in Britain. Harper Collins, 2006.

[15] J.C. Biesmeijer, S.P.M. Roberts, M. Reemer, R. Ohlemüller, M. Edwards, T. Peeters, A.P. Schaffers, S.G. Potts, R. Kleukers, C.D. Thomas, et al. Parallel declines in pollinators and insect-pollinated plants in Britain and the Netherlands. Science, 313(5785):351-354, 2006.

[16] R. Bommarco, O. Lundin, H.G. Smith, and M. Rundlöf. Drastic historic shifts in bumble-bee community composition in Sweden. Proceedings of the Royal Society B, 279(1727):309-315, 2012.

[17] J.S. Borrell. Rapid assessment protocol for pollen settling velocity: implications for habitat fragmentation. Bioscience Horizons, 5:hzs002, 2012.

[18] J. Bryden, R.J. Gill, R.A.A. Mitton, N.E. Raine, and V.A.A. Jansen. Chronic sublethal stress causes bee colony failure. Ecology Letters, 16(12):1463-1469, 2013.

[19] C. Carvell, A.F.G. Bourke, J.L. Osborne, and M.S. Heard. Effects of an agri-environment scheme on bumblebee reproduction at local and landscape scales. Basic and Applied Ecology, 16(6):519-530, 2015.

[20] E.E. Crone and N.M. Williams. Bumble bee colony dynamics: quantifying the importance of land use and floral resources for colony growth and queen production. Ecology Letters, 19(4):460-468, 2016.

[21] D.T. Crouse, L.B. Crowder, and H. Caswell. A stage-based population model for loggerhead sea turtles and implications for conservation. Ecology, 68(5):1412-1423, 1987.

[22] J.M. Cushing. Integrodifferential equations and delay models in population dynamics, Vol. 20. Springer Science \& Business Media, 2013.

[23] M.J. Duchateau. Agonistic behaviours in colonies of the bumblebee Bombus terrestris. Journal of Ethology, $7(2): 141-151,1989$.

[24] M.J. Duchateau and H.H.W. Velthuis. Development and reproductive strategies in Bombus terrestris colonies. Behaviour, 107(3):186-207, 1988.

[25] M.J. Duchateau, H.H.W. Velthuis, and J.J. Boomsma. Sex ratio variation in the bumblebee Bombus terrestris. Behavioral Ecology, 15(1):71-82, 2004.

[26] A. Fauser-Misslin, B.M. Sadd, P. Neumann, and C. Sandrock. Influence of combined pesticide and parasite exposure on bumblebee colony traits in the laboratory. Journal of Applied Ecology, 51(2):450-459, 2014. 
[27] H. Feltham, K. Park, and D. Goulson. Field realistic doses of pesticide imidacloprid reduce bumblebee pollen foraging efficiency. Ecotoxicology, 23(3):317-323, 2014.

[28] C. Fontaine, I. Dajoz, J. Meriguet, and M. Loreau. Functional diversity of plant-pollinator interaction webs enhances the persistence of plant communities. PLoS Biol, 4(1):e1, 2005.

[29] L.A. Garibaldi, I. Steffan-Dewenter, R. Winfree, M.A. Aizen, R. Bommarco, S.A. Cunningham, C. Kremen, L.G. Carvalheiro, L.D. Harder, O. Afik, et al. Wild pollinators enhance fruit set of crops regardless of honey bee abundance. Science, 339(6127):1608-1611, 2013.

[30] R.J. Gill, O. Ramos-Rodriguez, and N.E. Raine. Combined pesticide exposure severely affects individual- and colony-level traits in bees. Nature, 491(7422):105-108, 2012.

[31] R.J. Gill, O. Ramos-Rodriguez, and N.E. Raine. Combined pesticide exposure severely affects individual-and colony-level traits in bees: Supplementary material. Nature, 491, 2012.

[32] H.C.J. Godfray, T. Blacquiere, L.M. Field, R.S. Hails, S.G. Potts, N.E. Raine, A.J. Vanbergen, and A.R. McLean. A restatement of recent advances in the natural science evidence base concerning neonicotinoid insecticides and insect pollinators. Proceeding of the Royal Society B, 282(1818):20151821, 2015.

[33] K. Gopalsamy. Stability and oscillations in delay differential equations of population dynamics. Vol. 74. Springer Science \& Business Media, 2013.

[34] D. Goulson. Bumblebees: behaviour, ecology, and conservation. Oxford University Press on Demand, 2010.

[35] D. Goulson. Review: An overview of the environmental risks posed by neonicotinoid insecticides. Journal of Applied Ecology, 50(4):977-987, 2013.

[36] D. Goulson, J. Peat, J.C. Stout, J. Tucker, B. Darvill, L.C. Derwent, and W.O.H. Hughes. Can allotheism in workers of the bumblebee, Bombus terrestris, be explained in terms of foraging efficiency? Animal Behavior, 64:123-130, 2002.

[37] J.G. Holland, F.S. Guidat, and A.F.G. Bourke. Queen control of a key life-history event in a eusocial insect. Biology Letters, 9(3):20130056, 2013.

[38] G.E. Hutchinson. Circular causal systems in ecology. Annals of the New York Academy of Sciences, 50(4):221$246,1948$.

[39] F. Kappel. Spline approximation for autonomous nonlinear functional differential equations. Nonlinear Analysis: Theory, Methods \& Applications, 10(5):503-513, 1986.

[40] F. Kappel and W. Schappacher. Autonomous nonlinear functional differential equations and averaging approximations. Nonlinear Analysis: Theory, Methods $\& 5$ Applications, 2(4):391-422, 1978.

[41] D.S. Khoury, A.B. Barron, and M.R. Myerscough. Modelling food and population dynamics in honey bee colonies. PloS one, 8(5):e59084, 2013.

[42] D. Kleijn, R. Winfree, I. Bartomeus, L.G. Carvalheiro, M. Henry, R. Isaacs, A. Klein, C. Kremen, L.K. M'gonigle, R. Rader, et al. Delivery of crop pollination services is an insufficient argument for wild pollinator conservation. Nature Communications 6, 2015.

[43] A. Klein, B.E. Vaissiere, J.H. Cane, I. Steffan-Dewenter, S.A. Cunningham, C. Kremen, and T. Tscharntke. Importance of pollinators in changing landscapes for world crops. Proceedings of the Royal Society of London B: Biological Sciences, 274(1608):303-313, 2007.

[44] M. Kot. Elements of mathematical ecology. Cambridge University Press, 2001. 
[45] C. Lopez-Vaamonde, N.E. Raine, J.W. Koning, R.M. Brown, J.J.M. Pereboom, T.C. Ings, O. RamosRodriguez, W.C. Jordan, and A.F.G. Bourke. Lifetime reproductive success and longevity of queens in an annual social insect. Journal of Evolutionary Biology, 22(5):983-996, 2009.

[46] O. Lundin, M. Rundlöf, H.G. Smith, I. Fries, and R. Bommarco. Neonicotinoid insecticides and their impacts on bees: a systematic review of research approaches and identification of knowledge gaps. PloS One, 10(8):e0136928, 2015.

[47] S. Macevicz and G. Oster. Modeling social insect populations ii: optimal reproductive strategies in annual eusocial insect colonies. Behavioral Ecology and Sociobiology, 1(3):265-282, 1976.

[48] W.F. Morris and D.F. Doak. Quantitative conservation biology: theory and practice of population viability analysis. Sinauer Associates, 2002.

[49] C.B. Müller, J.A. Shykoff, and G.H. Sutcliffe. Life history patterns and opportunities for queen-worker conflict in bumblebees (hymenoptera: Apidae). Oikos, 65(2):242-248, 1992.

[50] J. Ollerton, R. Winfree, and S. Tarrant. How many flowering plants are pollinated by animals? Oikos, 120(3):321-326, 2011.

[51] G. Oster. Modeling social insect populations. i. ergonomics of foraging and population growth in bumblebees. American Naturalist, 110(972):215-245, 1976.

[52] J. Peat and D. Goulson. Effects of experience and weather on foraging rate and pollen versus nectar collection in the bumblebee, Bombus terrestris. Behavioral Ecology and Sociobiology, 58(2):152-156, 2005.

[53] J.J.M. Pereboom. The composition of larval food and the significance of exocrine secretions in the bumblebee Bombus terrestris. Insectes Sociaux, 47(1):11-20, 2000.

[54] J.J.M. Pereboom, H.H.W. Velthuis, and M.J. Duchateau. The organisation of larval feeding in bumblebees (Hymenoptera: Apidae) and its significance to caste differentiation. Insectes Sociaux, 50(2):127-133, 2003.

[55] K. Poitrineau, O. Mitesser, and H.J. Poethke. Workers, sexuals, or both? optimal allocation of resources to reproduction and growth in annual insect colonies. Insectes Sociaux, 56(2):119-129, 2009.

[56] S.G. Potts, J.C. Biesmeijer, C. Kremen, P. Neumann, O. Schweiger, and W.E. Kunin. Global pollinator declines: trends, impacts and drivers. Trends in Ecology $\&$ Evolution, 25(6):345-353, 2010.

[57] M.F. Ribeiro. Growth in bumble bee larvae: relation between development time, mass, and amount of pollen ingested. Canadian Journal of Zoology, 72(11):1978-1985, 1994.

[58] M.F. Ribeiro, H.H.W. Velthuis, M.J. Duchateau, and I. Van der Tweel. Feeding frequency and caste differentiation in Bombus terrestris larvae. Insectes Sociaux, 46(4):306-314, 1999.

[59] P. Röseler. Unterschiede in der kastendetermination zwischen den hummelarten Bombus hypnorum und Bombus terrestris (Differences in the caste determination between the bumblebee species Bombus hypnorum and Bombus terrestris. Zeitschrift für Naturforschung B, 25(5):543-548, 1970.

[60] M. Rundlöf, A.S. Persson, H.G. Smith, and R. Bommarco. Late-season mass-flowering red clover increases bumble bee queen and male densities. Biological Conservation, 172:138-145, 2014.

[61] H. Smith. An introduction to delay differential equations with applications to the life sciences. Springer, 2011.

[62] G.H. Sutcliffe and R.C. Plowright. The effects of pollen availability on development time in the bumble bee Bombus terricola K.(Hymenoptera: Apidae). Canadian Journal of Zoology, 68(6):1120-1123, 1990. 
[63] N.M. Williams, J. Regetz, and C. Kremen. Landscape-scale resources promote colony growth but not reproductive performance of bumble bees. Ecology, 93(5):1049-1058, 2012.

[64] R. Winfree, I. Bartomeus, and D.P. Cariveau. Native pollinators in anthropogenic habitats. Annual Review of Ecology, Evolution, and Systematics, 42(1):1, 2011.

[65] T.J. Wood, J.M. Holland, W.O.H. Hughes, and D. Goulson. Targeted agri-environment schemes significantly improve the population size of common farmland bumblebee species. Molecular Ecology, 24(8):1668-1680, 2015 . 